\title{
SELEÇÃO DE MICRO-ORGANISMOS PARA DIFERENTES SISTEMAS ENZIMÁTICOS
}

\author{
L. TIGGEMEN ${ }^{1}$, N. GRIEBELER ${ }^{1}$, V. DE BORTOLI ${ }^{1}$, A. ASTOLFI $^{1}$, N. $^{2}$ \\ DARONCH $^{1}$, A. SCHUMANN ${ }^{1}$, L. NOSKOSKI ${ }^{1}$, C. STEFENS ${ }^{1}$, R. L. CANSIAN ${ }^{1}$, G. \\ TONIAZZO ${ }^{1}$ e J. ZENI ${ }^{1}$
}

${ }^{1}$ Universidade Regional Integrada do Alto Uruguai e Missões, Departamento de Engenharia de Alimentos

Email para contato: liditiggemann@hotmail.com

\begin{abstract}
RESUMO- A exploração da biodiversidade na busca de novos microorganismos produtores de enzimas como amilases, proteases, celulases e pectinases pode vir a contribuir para a descoberta de micro-organismos que produzam enzimas com alto teor de rendimento, estabilidade, especificidade, de produção e recuperação de baixo custo. Neste sentido, o presente trabalho teve como objetivo selecionar fungos filamentosos, potencialmente capazes de produzir amilase, proteases, celulases e pectinases. A selecção de microorganismos com atividade enzimática foi realizada por plaqueamento dos isolados em meio sólido com diferentes fontes de carbono e medida do diâmetro de degradação. O screening resultou em 180 micro-organismos selecionados, destes, $12,2 \%$ produziram proteases, $17,2 \%$ amilases, $27,2 \%$ celulases, e $32,7 \%$ pectinases e $10,7 \%$ não produziram nenhuma destas enzimas. Dos 180 isolados, 2 apresentaram atividade para todas as enzimas avaliadas, este amplo espectro de atividade indica um elevado potencial de aplicação destes isolados.
\end{abstract}

\section{INTRODUÇÃO}

O uso de enzimas nas indústrias possibilita o desenvolvimento de processos tecnológicos tão eficientes quanto aos realizados pela natureza e sem causar riscos ambientais. Consequientemente, a demanda mundial destas enzimas tem crescido anualmente, sendo mais de $90 \%$ do seu comércio efetuado pelos Estados Unidos, Europa e Japão (Gupta et al., 2004).

Amilases, proteases e celulases podem ser originárias de plantas, animais e microorganismos. As microbianas sendo produzidas principalmente por bactérias e fungos, representam a mais ampla classe de enzimas aplicadas no ramo da biotecnologia e química orgânica (Jaeger e Reetz, 1998).

$\mathrm{O}$ isolamento e seleção de micro-organismos podem facilitar a descoberta de novas enzimas com características desejáveis (estabilidade a altas temperaturas e amplas faixas de $\mathrm{pH}$, alta especificidade. Além disso, pode abrir promissoras perspectivas científicas e comerciais (Silva, 2002). 
O crescente aumento das pesquisas na área da enzimologia estimula a descoberta de novos micro-organismos a fim de aumentar a produtividade, a especificidade e a estabilidade das enzimas (Geok et al., 2003). Com base no que foi abordado anteriormente, o objetivo geral deste trabalho foi selecionar micro-organismos com habilidade em produzir enzimas amilases, celulases e proteases.

\section{METODOLOGIA}

\subsection{Screening de micro-organismos}

Os micro-organismos utilizados foram obtidos da coleção previamente isolada de micro-organismos do Laboratório de Biotecnologia de Alimentos, do curso de Engenharia de Alimentos da URI, Campus de Erechim. A coleção de micro-organismos utilizados foi obtida de óleo de oliva, requeijão, queijo minas, extrato de tomate, óleo de soja, folhas, frutas, nata, creme de leite, carne, maionese e também de amostras de farelo de soja deterioradas e contaminações existentes em meios de cultura. $\mathrm{O}$ isolamento dos micro-organismos foi feito por sub-cultivos em placas de Petri contendo meio PDA (agar batata dextrose) incubado a $25^{\circ} \mathrm{C}$ por 3 dias, até o isolamento completo das colônias. Estes micro-organismos previamente isolados foram testados como produtores de enzimas em placas com meio sólido específico para cada enzima. Em todos os sistemas o diâmetro do halo de degradação do substrato será medido em milímetros, subtraindo-se o diâmetro do crescimento micelial. Ou seja, será avaliado o diâmetro do halo, e não o diâmetro da colônia.

\section{Atividade amilolítica in vitro}

Os isolados foram cultivados em meio mínimo, o qual teve a glicose substituída por amido, incubados por sete dias a $25^{\circ} \mathrm{C}$. Decorridos sete dias de cultivo dos isolados no meio, procedeu-se à revelação do halo de degradação do amido. Para isso, foram vertidos $2 \mathrm{~mL}$ de solução lugol sobre a superfície do meio de cultura. Após 10 minutos, a solução lugol será descartada e a atividade amilolítica detectada pela formação de halo claro circundado por uma zona azulada.

\section{Atividade celulolítica in vitro}

Os isolados foram cultivados em agar carboximetilcelulose. Estes foram incubados por sete dias a $25^{\circ} \mathrm{C}$. Após esse período, foram adicionados $10 \mathrm{~mL}$ de solução corante de vermelho congo em tampão Tris $\mathrm{HCl}$ 0,05M, pH 8,5. Após 30 minutos a solução foi descartada e as culturas serão lavadas com $5 \mathrm{~mL}$ de solução de $\mathrm{NaCl} 0,5 \mathrm{M}$ neste mesmo tampão, para a revelação do halo claro e estreito de degradação da celulose ao redor da colônia.

\section{Atividade proteolítica in vitro}

Para detectar a atividade proteolítica, foi utilizado o meio nutriente ágar, contendo gelatina como substrato. As placas serão incubadas durante sete dias a $25^{\circ} \mathrm{C}$. Após o período de incubação dos isolados neste meio, foi adicionado sobre o mesmo uma solução saturada de sulfato de amônio, cujo precipitado tornou o ágar mais opaco e acentuará as zonas claras ao redor das colônias, onde a gelatina será degradada. 


\section{RESULTADOS}

\subsection{Screening de micro-organismos}

Neste estudo foram previamente isolados 180 fungos filamentosos provenientes de diversas fontes como óleo de oliva, requeijão, queijo minas, extrato de tomate, óleo de soja, folhas, frutas, nata, creme de leite, carne, maionese e também de amostras de farelo de soja deterioradas e contaminações existentes em meios de cultura.

Levando em consideração a possibilidade da obtenção de micro-organismos bons produtores de enzimas, as cepas isoladas foram utilizadas no screening em placas com meio mínimo contendo meios específicos para a identificação de atividade amilolítica, proteolítica e celulolítica. Em todos os sistemas o diâmetro do halo de degradação do substrato foi medido em milímetros, subtraindo-se o diâmetro do crescimento micelial. Ou seja, foi avaliado o diâmetro do halo, e não o diâmetro da colônia.

\subsection{Atividade amilolítica}

Os resultados da seleção de micro-organismos para a produção de amilases encontram-se na Tabela 1. O diâmetro dos halos dos diferentes isolados com atividade amilolítica variaram entre 2,2 e 11,06 mm, sem considerar o diâmetro da colônia. Foram considerados com maior potencial de atividade amilolítica 4 isolados (I48, I50, I19 e I210) com diâmetro de halo acima de 7,08 (média mais desvio padrão) entre todos os produtores de amilase.

Tabela 1- Tamanho do halo ( $\mathrm{mm}$ ) dos diferentes micro-organismos isolados com atividade amilolítica.

\begin{tabular}{cccc}
\hline $\begin{array}{c}\text { Micro-organismos } \\
\text { isolados }\end{array}$ & $\begin{array}{c}\text { Diametro do } \\
\text { halo }(\mathbf{m m})\end{array}$ & $\begin{array}{c}\text { Micro-organismos } \\
\text { isolados }\end{array}$ & $\begin{array}{c}\text { Diametro do halo } \\
(\text { mm })\end{array}$ \\
\hline I48 (Aspergillus sp. $)$ & $11,06 \pm 1,1^{\mathrm{a}}$ & $\mathrm{I} 34$ & $4,4 \pm 0,4^{\mathrm{e}}$ \\
I50 (Penicillium sp. $)$ & $8 \pm 0,8^{\mathrm{b}}$ & $\mathrm{I} 38$ & $3,8 \pm 0,4^{\mathrm{e}}$ \\
I19 (Aspergillus sp.) & $7,6 \pm 0,6^{\mathrm{c}}$ & $\mathrm{I} 73$ & $3,08 \pm 0,3^{\mathrm{e}}$ \\
I210 (Aspergillus sp. $)$ & $7,4 \pm 0,7^{\mathrm{c}}$ & $\mathrm{I} 17$ & $3,8 \pm 0,4^{\mathrm{e}}$ \\
I11 & $5,8 \pm 0,6^{\mathrm{d}}$ & $\mathrm{I} 214$ & $3,06 \pm 0,5^{\mathrm{ef}}$ \\
I23 & $5,8 \pm 0,6^{\mathrm{d}}$ & $\mathrm{I} 54$ & $3 \pm 0,3^{\mathrm{f}}$ \\
I211 & $5,8 \pm 0,6^{\mathrm{d}}$ & $\mathrm{I} 68$ & $3 \pm 0,3^{\mathrm{f}}$ \\
I27 & $5,6 \pm 0,6^{\mathrm{d}}$ & $\mathrm{I} 71$ & $3 \pm 0,3^{\mathrm{f}}$ \\
I213 & $5,4 \pm 0,8^{\mathrm{d}}$ & $\mathrm{I} 10$ & $3 \pm 0,3^{\mathrm{f}}$ \\
I70 & $5,12 \pm 0,5^{\mathrm{d}}$ & $\mathrm{I} 205$ & $2,4 \pm 0,2^{\mathrm{g}}$ \\
I67 & $5,1 \pm 0,5^{\mathrm{d}}$ & $\mathrm{I} 3$ & $2,2 \pm 0,2^{\mathrm{g}}$ \\
\hline
\end{tabular}

\subsection{Atividade celulolítica}

A atividade celulolítica foi observada em 39 isolados, sendo que destes, 8 isolados (I34, I130, I212, I30, I70, I26 e I23) apresentaram halos maiores a 13,86 mm (média mais desvio padrão) (Tabela 2). 
Tabela 2- Tamanho do halo ( $\mathrm{mm}$ ) dos diferentes isolados com atividade celulolítica.

\begin{tabular}{cccc}
\hline $\begin{array}{c}\text { Micro-organismos } \\
\text { isolados }\end{array}$ & $\begin{array}{c}\text { Diametro do } \\
\text { halo }(\mathbf{m m})\end{array}$ & $\begin{array}{c}\text { Micro-organismos } \\
\text { isolados }\end{array}$ & $\begin{array}{c}\text { Diametro do halo } \\
(\mathbf{m m})\end{array}$ \\
\hline I34 (Aspergillus sp.) & $17,4 \pm 1,8^{\mathrm{a}}$ & $\mathrm{I} 205$ & $7,2 \pm 0,7^{\mathrm{g}}$ \\
I130 (Penicillium sp.) & $16,4 \pm 1,6^{\mathrm{ab}}$ & $\mathrm{I} 10$ & $7,2 \pm 0,7^{\mathrm{g}}$ \\
I212 (Aspergillus sp.) & $16,2 \pm 1,6^{\mathrm{ab}}$ & $\mathrm{I} 210$ & $7,08 \pm 0,7^{\mathrm{g}}$ \\
I30 (Aspergillus sp.) & $15,8 \pm 1,6^{\mathrm{bc}}$ & $\mathrm{I} 223$ & $7,1 \pm 0,7^{\mathrm{fg}}$ \\
I70 (Penicillium sp.) & $15,2 \pm 1,4^{\mathrm{c}}$ & $\mathrm{I} 213$ & $6,6 \pm 0,6^{\mathrm{g}}$ \\
I26 (Aspergillus sp.) & $15,2 \pm 1,5^{\mathrm{c}}$ & $\mathrm{I} 44$ & $6 \pm 0,6^{\mathrm{gh}}$ \\
I73 (Penicillium sp.) & $14,8 \pm 1,4^{\mathrm{c}}$ & $\mathrm{I} 48$ & $6 \pm 0,5^{\mathrm{gh}}$ \\
I23 (Aspergillus sp.) & $14,4 \pm 1,4^{\mathrm{cd}}$ & $\mathrm{I} 33$ & $5 \pm 0,4^{\mathrm{h}}$ \\
I103 & $13 \pm 1,2^{\mathrm{d}}$ & $\mathrm{I} 80$ & $4,6 \pm 0,4^{\mathrm{h}}$ \\
I203 & $11,8 \pm 1,1^{\mathrm{e}}$ & $\mathrm{I} 211$ & $4,6 \pm 0,4^{\mathrm{h}}$ \\
I215 & $11,12 \pm 1,1^{\mathrm{e}}$ & $\mathrm{I} 224$ & $3,2 \pm 0,3^{\mathrm{i}}$ \\
I42 & $10 \pm 1,0^{\mathrm{ef}}$ & $\mathrm{I} 82$ & $3,12 \pm 0,3^{\mathrm{i}}$ \\
I221 & $9,8 \pm 1,0^{\mathrm{f}}$ & $\mathrm{I} 41$ & $3 \pm 0,2^{\mathrm{i}}$ \\
I225 & $9,12 \pm 0,9^{\mathrm{f}}$ & $\mathrm{I} 204$ & $2,4 \pm 0,2^{\mathrm{j}}$ \\
I216 & $8,2 \pm 0,8^{\mathrm{f}}$ & $\mathrm{I} 9$ & $2,4 \pm 0,2^{\mathrm{j}}$ \\
I28 & $8 \pm 0,8^{\mathrm{f}}$ & $\mathrm{I} 15$ & $2,4 \pm 0,3^{\mathrm{j}}$ \\
I36 & $8 \pm 0,7^{\mathrm{f}}$ & $\mathrm{I} 1$ & $2,2 \pm 2,0^{\mathrm{j}}$ \\
I222 & $7,8 \pm 0,8^{\mathrm{f}}$ & $\mathrm{I} 43$ & $2 \pm 0,2^{\mathrm{j}}$ \\
I31 & $7,8 \pm 0,8^{\mathrm{f}}$ & $\mathrm{I} 20$ & $1 \pm 0,1^{1}$ \\
I11 & $7,4 \pm 0,8^{\mathrm{g}}$ & ----- & ---- \\
\hline
\end{tabular}

*--- não houve isolado

\subsection{Atividade Proteolítica}

Dentre os micro-organismos selecionados com atividade proteolítica (Tabela 3), apenas 5 isolados (I215, I58, I32, I216 e I222) apresentaram diâmetro de halo acima de 10,62mm (média mais desvio padrão) entre todos os produtores.

Tabela 3- Tamanho do halo ( $\mathrm{mm}$ ) dos diferentes isolados com atividade proteolítica.

\begin{tabular}{|c|c|c|c|}
\hline $\begin{array}{c}\text { Micro-organismos } \\
\text { isolados }\end{array}$ & $\begin{array}{c}\text { Diametro do } \\
\text { halo ( } \mathrm{mm})\end{array}$ & $\begin{array}{c}\text { Micro-organismos } \\
\text { isolados }\end{array}$ & $\begin{array}{c}\text { Diametro do halo } \\
(\mathrm{mm})\end{array}$ \\
\hline I215 (Penicillium sp.) & $23,12 \pm 2,4^{\mathrm{a}}$ & $\mathrm{I} 31$ & $3 \pm 0,4^{i}$ \\
\hline I58 (Penicillium sp.) & $19,2 \pm 1,8^{b}$ & $\mathrm{I} 34$ & $3 \pm 0,3^{i}$ \\
\hline I32 (Aspergillus sp.) & $12,6 \pm 1,3^{\mathrm{c}}$ & $\mathrm{I} 36$ & $3 \pm 0,3^{\mathrm{i}}$ \\
\hline I216 (Aspergillus sp.) & $11,54 \pm 1,1^{\mathrm{c}}$ & $\mathrm{I} 26$ & $3 \pm 0,3^{\mathrm{i}}$ \\
\hline I222 (Aspergillus sp.) & $11,4 \pm 1,3^{\mathrm{c}}$ & I65 & $3 \pm 0,3^{\mathrm{i}}$ \\
\hline I211 & $9,12 \pm 0,8^{\mathrm{d}}$ & $\mathrm{I} 219$ & $2,8 \pm 0,2^{\mathrm{ij}}$ \\
\hline $\mathrm{I} 217$ & $9,06 \pm 0,9^{\mathrm{d}}$ & $\mathrm{I} 210$ & $2,6 \pm 0,2^{\mathrm{j}}$ \\
\hline $\mathrm{I} 76$ & $9 \pm 0,9^{\mathrm{d}}$ & I68 & $2 \pm 0,2^{1}$ \\
\hline I 218 & $7,8 \pm 0,8^{\mathrm{e}}$ & I84 & $2 \pm 0,2^{1}$ \\
\hline $\mathrm{I} 213$ & $6,4 \pm 0,6^{\mathrm{ef}}$ & $\mathrm{I} 25$ & $2 \pm 0,2^{1}$ \\
\hline $\mathrm{I} 220$ & $5,1 \pm 0,5^{\mathrm{g}}$ & $\mathrm{I} 40$ & $2 \pm 0,2^{1}$ \\
\hline $\mathrm{I} 30$ & $5,1 \pm 0,5^{\mathrm{g}}$ & I44 & $2 \pm 0,2^{1}$ \\
\hline $\mathrm{I} 15$ & $4,12 \pm 0,4^{\mathrm{h}}$ & I54 & $2 \pm 0,2^{1}$ \\
\hline I11 & $3,8 \pm 0,4^{\mathrm{h}}$ & I81 & $1 \pm 0,1^{\mathrm{m}}$ \\
\hline $\mathrm{I} 27$ & $3,8 \pm 0,4^{\mathrm{h}}$ & $\mathrm{I} 23$ & $1 \pm 0,1^{\mathrm{m}}$ \\
\hline $\mathrm{I} 2$ & $3,6 \pm 0,6^{\mathrm{hi}}$ & ------ & \\
\hline
\end{tabular}

*--- não houve isolado 


\subsection{Isolados com Amplo Espectro de Atividade Enzimática}

Dos 180 isolados, dois apresentaram atividade para todas as enzimas avaliadas, I211 (Penicillium sp.) e I213 (Paecelomyces sp.). Embora a atividade destes não seja alta nos diferentes sistemas, este amplo espectro de atividade indica um alto potencial destes isolados, principalmente para utilização no tratamento de resíduos, uma vez que um único micro-organismo pode degradar diferentes substratos presentes no resíduo.

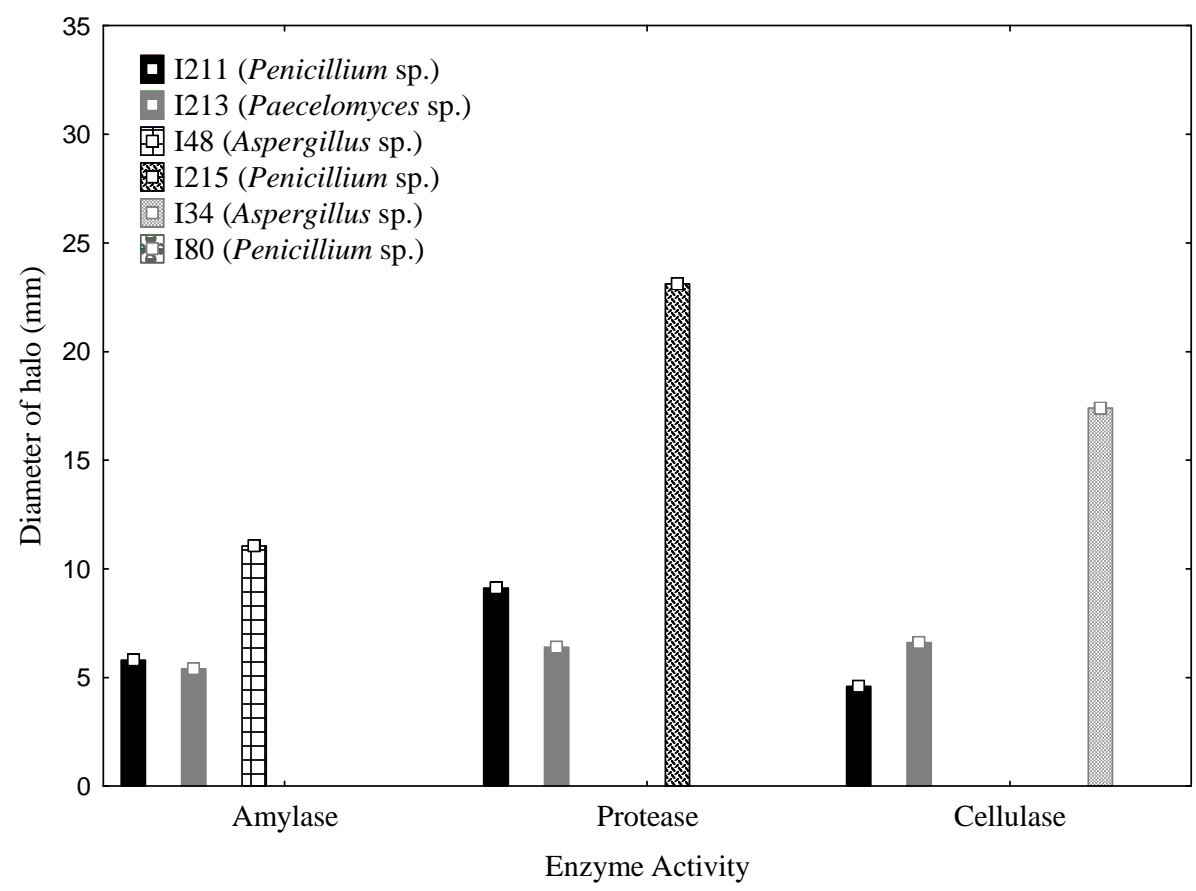

Figura 1- Espectro de atividade enzimática dos isolados I211 (Penicillium sp.) e I213 (Paecelomyces sp.) em comparação com os isolados de maior atividade de cada uma das enzimas avaliadas.

Isolados de Crinipellis perniciosa, obtidos a partir de cacaueiro (Theobromae cacao), cupuaçuzeiro (T. grandiflorum) e solanáceas silvestres foram testados quanto à capacidade de produzirem enzimas extracelulares que degradam celulose, amido, lipídios, proteínas e lignina. A produção de todas as enzimas foi determinada em meios sólidos e representada por uma estimativa, baseada na intensidade de cor, ou pela avaliação do diâmetro dos halos formado nos meios. Foi detectada variabilidade entre os isolados na capacidade de produzir enzimas celulolíticas, amilases, lipases, polifenoloxidases, peroxidases e esterases. Quanto às enzimas proteolíticas, todos os isolados apresentaram alto nível de atividade, não sendo observada diferença no comportamento entre eles. Por outro lado, nenhum dos isolados produziu pectinase, urease e fosfataseácida (Bastos, 2005).

Em trabalho de Souza (2008) foi possível detectar a produção de celulases e proteases por todos os isolados fúngicos, $40 \%$ produziram amilases, $50 \%$ produziram fenoloxidases e $10 \%$ produziram pectinases. Peixoto (2006) testou 390 cepas no processo de triagem, foram selecionadas 45 cepas, consideradas potencialmente produtoras de amilases $(12,31 \%) ; 84(21,54 \%)$, produtoras de CMCase; $46(11,79 \%)$, produtoras de celulases e $35(8,97 \%)$, produtoras de gomas. 


\section{CONCLUSÃO}

Dos 180 isolados, $12,2 \%$ produziram amilases, 17,2\% proteases, 27,2\% produziram celulases, $32,7 \%$ produziram pectinases e $10,7 \%$ não produziram nenhuma destas enzimas. De todos os micro-organismos testados, 2 micro-organismos codificados I211 (Penicillium sp.) e I213 (Paecelomyces sp.) apresentaram atividade para todas as enzimas avaliadas. Embora a atividade destes não seja alta nos diferentes sistemas, este amplo espectro de atividade indica um alto potencial destes isolados, principalmente para utilização no tratamento de resíduos, uma vez que um único microorganismo pode degradar diferentes substratos presentes no resíduo.

\section{AGRADECIMENTOS}

Os autores agradecem ao CNPq, CAPESe FAPERGS pelo suporte financeiro deste trabalho e bolsas de estudo.

\section{REFERÊNCIAS}

BASTOS, C. N. Produção de enzimas extracelulares por Crinipellis perniciosa. Fitopatol. bras., v. 30, p. 286-288, 2005.

GEOK, L. P.; RAZAK, C. N. A.; ABD RAHMAN, R. N. Z.; BASRI, M.; SALLEH, A. B. Isolation and screening of an extracellular organic solvent-tolerant protease producer. Biochem. Eng. J., v. 13, p. 73-77, 2003.

GUPTA, R.; GUPTA, N.; RATHI, P. Bacterial lipases: an overview of production, purification and biochemical properties. App Microbiol. Biotechnol., v. 64, p. 763781, 2004.

JAEGER, K. E; REETZ, M. T. Microbial lipases form versatile tools for biotechnology. Biotechnol., v. 16, p. 396-403, 1998.

PEIXOTO, A. B. Estudo da produção de enzimas e gomas de leveduras selvagens coletadas em diversas regiões do Brasil. 84 f. Dissertação de Mestrado em Engenharia de Alimentos - Faculdade de Engenharia de Alimentos, Universidade Estadual de Campinas, Campinas. 2006.

SILVA, D.; MARTINS, E. S.; SILVA, GOMES, R. Pectinase production by Penicillium viridicatum RFC3 by solid state fermentation using agricultural wastes and agro-industrial by-products. Braz. J. Microbiol. vol.33, p.318-324, 2002.

SOUZA, H. Q.; OLIVEIRA, L. A.; ANDRADE, J. S.. Seleção de Basidiomycetes da Amazônia para produção de enzimas de interesse biotecnológico. Ciênc. Tecnol. Aliment., v. 28, p. 116-124, 2008. 